\title{
Global Weak Solutions for an Incompressible Charged Fluid with Multi-Scale Couplings: Initial-Boundary Value Problem
}

\author{
Joseph W. Jerome ${ }^{\mathrm{a}, 1}$ and Riccardo Sacco ${ }^{\mathrm{b}, 2}$ \\ ${ }^{a}$ Department of Mathematics, Northwestern University, \\ Evanston, IL 60208-2730, USA \\ bDipartimento di Matematica "F. Brioschi", Politecnico di Milano, \\ via Bonardi 9, 20133 Milano, Italy
}

\begin{abstract}
The Cauchy problem for the Poisson-Nernst-Planck/Navier-Stokes model was investigated by the first author in [Transport Theory Statist. Phys. 31 (2002), 333-366], where a local existence-uniqueness theory was demonstrated, based upon Kato's framework for examining evolution equations. In this article, the existence of a global weak solution is proved to hold for the model, in the case of the initial-boundary value problem. Connection of the above analysis to significant applications is addressed, including bio-hybrid devices in neuronal cell monitoring, bio-reactor devices in tissue engineering and microfluidic devices in Lab-On-Chip technology.
\end{abstract}

Key words: Navier-Stokes, Poisson-Nernst-Planck, initial boundary problem for hybrid systems, Rothe's method, slip boundary condition

\section{Introduction}

In this article, we rigorously investigate a general class of mathematical models originating in bio-electronic applications. When fully developed, these models are intended to account for the electrical, fluid-mechanical and bio-chemical

Email addresses: jwj@math.northwestern.edu (Joseph W. Jerome), riccardo.sacco@polimi.it (Riccardo Sacco).

1 Corresponding author. This author was supported by ONR/Darpa grant nr. LLCN00014-05-C-0241

2 This author was supported by the M.U.R.S.T. grant nr. 2006013187-003 
phenomena simultaneously occurring in complex bio-hybrid systems like biochips $[9,21]$, bio-reactors $[23,7]$ or microfluidic chambers in Lab-On-Chip technology $[5,16]$. The present article represents a continuation via analysis of earlier applied studies [17-19].

To accomplish the above objectives, we consider the nonlinearly coupled Poisson-Nernst-Planck/Navier-Stokes (PNP/NS) system of PDEs, suitably coupled with generation/reaction zero-order terms. The basic system was introduced by Rubinstein in [24]. Self-consistent charge transport is represented by the Poisson-Nernst-Planck system, and the fluid motion by a Navier-Stokes system with forcing terms. This model is capable of describing electro-chemical and fluid-mechanical transport throughout the cellular environment. This already engages a range of spatial and temporal scales. A local smooth theory based upon these equations was derived for the Cauchy problem in [14]. Chemical reactions in cellular metabolism can be studied by coupling this system to generation/reaction zero order terms, denoted by $R_{n}, R_{p}$ below. In this article, we analyze the initial/mixed-boundary value problem in $\Omega \subset \mathbf{R}^{m}$, and demonstrate the existence of a global weak solution. At the conclusion of the article, we describe a variety of current applications of the model, which is a significant one for a variety of fields. We are not aware of comparable mathematical results for coupled systems of incompressible charged flow. For fluid-structure systems which are modeled linearly, there are results; see [1] for a fluid/elasticity model analyzed via semigroup methods. In addition, the results of [20] extend semigroup methods to the Navier-Stokes system. The slip condition is discussed in [25] and regularity in [10]. Numerous results could be cited, and the omission of any related result is unintended.

\subsection{The Fluid/Transport System}

We begin by formulating the constitutive relations for the ionic current densities. They extend the usual relations, given in PNP theory, by the inclusion of velocity convection terms. If $\vec{v}$ is the velocity of the electrolyte, and the ionic concentrations are denoted by $n, p$, respectively, the current densities are:

$$
\begin{aligned}
\overrightarrow{J_{n}} & =e D_{n} \nabla n-e \mu_{n} n \nabla \phi-e \vec{v} n \\
\overrightarrow{J_{p}} & =-e D_{p} \nabla p-e \mu_{p} p \nabla \phi+e \vec{v} p .
\end{aligned}
$$

Here, $J_{n}, J_{p}$ are the anion and cation current densities, with corresponding (constant) diffusion and mobility coefficients, $D_{n}, D_{p}, \mu_{n}, \mu_{p}$, respectively. The charge modulus is given by $e$, and $\phi$ is the electric potential. The Poisson equation, given shortly, describes the coupling. The enhanced PNP system is then given by, with $\epsilon$ the dielectric constant: 


$$
\begin{aligned}
\frac{\partial n}{\partial t}-\frac{1}{e} \nabla \cdot \vec{J}_{n} & =-R_{n} \\
\frac{\partial p}{\partial t}+\frac{1}{e} \nabla \cdot \vec{J}_{p} & =-R_{p} \\
\vec{E} & =-\nabla \phi \\
\nabla \cdot(\epsilon \nabla \phi) & =e(n-p-d) \quad \text { (Poisson equation) }
\end{aligned}
$$

In the above, the 'fixed' charge concentration is denoted by $d$; though not mobile, it is an input which may change in time. The Einstein relations are employed: $D_{n}=\left(k T_{0} / e\right) \mu_{n}, D_{p}=\left(k T_{0} / e\right) \mu_{p}$. Here, $T_{0}$ is the ambient temperature; $k$ denotes Boltzmann's constant. For convenience, $R_{n}, R_{p}$ are assumed bounded and continuous in their arguments $\vec{v}, n, p$.

The velocity of the electrolyte is determined by the Navier-Stokes equations:

$$
\begin{aligned}
& \rho\left(\vec{v}_{t}+\vec{v} \cdot \nabla \vec{v}\right)-\eta \Delta \vec{v}=-\nabla P_{f}-e(p-n) \nabla \phi \\
& \nabla \cdot \vec{v}=0
\end{aligned}
$$

where $\rho$ is the constant (mass) density of the electrolyte, $P_{f}$ denotes fluid pressure, and $\eta$ is the constant dynamic viscosity. Note that $d$ has been neglected in the electric 'volume force' term. We shall make use of the kinematic viscosity, $\nu_{*}=\eta / \rho$, in the statement of the mathematical model. The above system is found essentially in [24]. Note that the signs of $R_{n}, R_{p}$ are those adopted in semiconductor physics, but the explicit forms are not essential for this study.

\subsection{Boundary Conditions and Assumptions}

We shall refer to the incompressibility condition (8) as a divergence free property. Projections onto such functions will be introduced subsequently. This methodology follows the original idea of Leray, permitting a pressure-free formulation (see [26] for extensive discussion). Scalar or vector functions with components in $H^{1}$ will sometimes be termed of finite energy. We now discuss the boundary conditions for the system. We distinguish between $\vec{v}$ and the remaining variables. For the latter, the boundary of $\Omega$ decomposes into two components: $\Sigma_{1}$, which is a Dirichlet boundary component, and $\Sigma_{2}$, which serves as a zero flux boundary component. So-called 'slip' Dirichlet boundary values for $\vec{v}$ are imposed on all of $\partial \Omega$, with the stipulation that a nonnegative (outward) normal fluid velocity component condition is imposed on $\partial \Omega$.

- This stipulation, which is frequently not met in numerical simulation, is unnecessary if the Navier-Stokes system is replaced by the Stokes subsystem. 
More precisely, we assume the existence of functions $\vec{v}_{B}, \phi_{B}, n_{B}, p_{B}$, defined continuously from $[0, T]$ into appropriately smooth spaces (described in the following definition), such that the following requirements hold for the solution vector:

$$
\vec{v}_{\mid \partial \Omega}=\vec{v}_{B \mid \partial \Omega}, \phi_{\mid \Sigma_{1}}=\phi_{B \mid \Sigma_{1}}, n_{\mid \Sigma_{1}}=n_{B \mid \Sigma_{1}}, p_{\mid \Sigma_{1}}=p_{B \mid \Sigma_{1}}
$$

The zero flux conditions on $\Sigma_{2}$ required for $\nabla \phi, \nabla n, \nabla p$, are incorporated into the weak formulation of the solution in the definition to follow. We assume that the boundary of $\Omega$ is sufficiently regular that the classical trace formulas and Green's integration by parts formulas are valid; a related assumption is that the completion in any $H^{s}(\Omega)$ space of $C^{\infty}(\bar{\Omega})$ is all of $H^{s}$. Finally, we assume that the Poisson solver is $H^{2}$ regularizing for $L^{2}$ data. Note that this implies that the mixed boundary conditions imposed by $\phi_{B}$ are not completely arbitrary. Although it is possible to distinguish the Dirichlet boundary component on which $\phi$ is defined from that on which $n, p$ are defined, this distinction is not implemented here.

\subsection{Weak Solution Characterization}

Definition 1.1 The space-time domain is denoted by $\mathcal{D}=\Omega \times[0, T]$; the space-time domain of the boundary is denoted $\mathcal{D}_{b}=\partial \Omega \times[0, T]$. Let $s \geq$ $m / 2$ be prescribed. We denote by $\mathcal{H}$ the divergence free functions in the $m$ fold Cartesian product of $H^{1}(\Omega)$, by $\mathcal{H}^{s}$ the intersection of $\mathcal{H}$ with the $m$-fold Cartesian product of $H^{s}(\Omega)$, and by $\mathcal{H}_{0}^{s}$ the zero trace subspace of $\mathcal{H}^{s} .\left(\mathcal{H}^{s}\right)^{*}$ is the dual of the latter space. Analogously, $H_{0, \Sigma_{1}}^{s}$ denotes the $H^{s}$ functions with zero trace on $\Sigma_{1}$. Moreover, for each $0 \leq t \leq T, \vec{v}_{B}(\cdot, t)$ has components in $\mathcal{H}^{s} \cap L^{\infty} ; \phi_{B}(\cdot, t) \in H^{\max (s, 2)}$; and $n_{B}(\cdot, t), p_{B}(\cdot, t) \in H^{s}$. Each of the functions, $\vec{v}_{B}, n_{B}, p_{B}$, is required to possess time derivatives in $L^{2}(\mathcal{D})$ and (trace time derivatives) in $L^{2}\left(\mathcal{D}_{b}\right)$. Also, initial value functions $\vec{v}_{0}, n_{0}, p_{0}$, of finite energy, with $\vec{v}_{0}$ divergence free are given. The fixed charge $d$ is continuous from $[0, T]$ into $L^{2}$. We define the regularity class $\mathcal{C}=\{\vec{u}=(\vec{v}, \phi, n, p)\}$ by the explicit conditions:

$$
\begin{gathered}
\vec{v} \in L^{2}((0, T) ; \mathcal{H}), n \text { and } p \in L^{2}\left((0, T) ; H^{1}\right), \phi \in L^{2}\left((0, T) ; H^{2}\right), \\
\vec{u}_{t} \in L^{2}\left((0, T) ;\left(\mathcal{H}_{0}^{s}\right)^{*} \times\left(H_{0, \Sigma_{1}}^{s}\right)^{*} \times\left(H_{0, \Sigma_{1}}^{s}\right)^{*}\right) .
\end{gathered}
$$

A weak solution of the PNP/Navier-Stokes system is a vector $\vec{u} \in \mathcal{C}$ such that (9) holds, such that $\phi$ is related to $n, p$ via (6), and, for $a(\vec{v}, \vec{v}, \vec{\psi}):=$ $\int_{\Omega} \vec{v} \cdot \nabla \vec{v} \vec{\psi} d \xi$, and functions $\vec{\psi}, \omega_{n}, \omega_{p}$, continuous from $[0, T]$ into $\mathcal{H}_{0}^{s} \times H_{0, \Sigma_{1}}^{s} \times$ $H_{0, \Sigma_{1}}^{s}$, with time derivatives in $L^{2}(\mathcal{D})$, we have: 


$$
\begin{aligned}
& \int_{\mathcal{D}}\left[\rho \vec{v} \frac{\partial \vec{\psi}}{\partial t}-\eta \nabla \vec{v} \cdot \nabla \vec{\psi}\right] d \xi d t-\int_{0}^{T} \rho a(\vec{v}, \vec{v}, \vec{\psi}) d t-e \int_{\mathcal{D}}(p-n) \nabla \phi \cdot \vec{\psi} d \xi d t \\
& =\int_{\Omega \times\{T\}} \vec{v} \cdot \vec{\psi} d \xi-\int_{\Omega \times\{0\}} \overrightarrow{v_{0} \cdot \vec{\psi} d \xi} \\
& \int_{\mathcal{D}}\left[n \frac{\partial \omega_{n}}{\partial t}-D_{n} \nabla n \cdot \nabla \omega_{n}+\left(\frac{e D_{n}}{k T_{0}}\right) n \nabla \phi \cdot \nabla \omega_{n}+\vec{v} n \cdot \nabla \omega_{n}\right] d \xi d t \\
& =\int_{\Omega \times\{T\}} n \omega_{n} d \xi-\int_{\Omega \times\{0\}} n_{0} \omega_{n} d \xi+\int_{\mathcal{D}} R_{n} \omega_{p} d \xi d t \\
& \int_{\mathcal{D}}\left[p \frac{\partial \omega_{p}}{\partial t}-D_{p} \nabla p \cdot \nabla \omega_{p}-\left(\frac{e D_{p}}{k T_{0}}\right) p \nabla \phi \cdot \nabla \omega_{p}+\vec{v} p \cdot \nabla \omega_{p}\right] d \xi d t \\
& =\int_{\Omega \times\{T\}} p \omega_{p} d \xi-\int_{\Omega \times\{0\}} p_{0} \omega_{p} d \xi+\int_{\mathcal{D}} R_{p} \omega_{n} d \xi d t
\end{aligned}
$$

If the highlighted term is missing, the system reduces to the PNP/Stokes system. In the next section, we provide an equivalent mapping characterization for the weak solution:

$$
\vec{u}_{t}+U \vec{u}=0,
$$

in an appropriate distribution sense.

\section{The System Mapping $U$ and General Properties for Solutions}

It will be helpful to define the system mapping. Since Rothe's method of horizontal lines underlies the proof, this will also include a steady framework. This section will provide the necessary transition.

\subsection{The Mapping}

The use of the trilinear form $a(\vec{v}, \vec{w}, \cdot)$ requires the domain of the mapping to include two components for the fluid system, rather than one, as might initially be expected. We shall order the individual components as follows: $\vec{v}, \vec{w} ; n ; p$. The associated component mappings are denoted $V, \Phi, N, P$. The system mapping is denoted $U=(V, \Phi, N, P)$. We shall now recall the domain spaces for these mappings. Since the problem is dynamic, we are actually defining an invariant domain for each $t \in[0, T]$.

- The invariant spatial domain of the system mapping $U$ is the Hilbert space:

$$
\mathcal{U}=\mathcal{H} \times \mathcal{H} \times H^{1} \times H^{1} .
$$

We begin by specifying the simplest of the mappings: $\Phi$ is an inverse mapping defined from the mixed boundary-value problem for (6). Its natural domain 
of definition is $L^{2} \times L^{2}$, so that its natural restriction includes elements taken from the two final spaces indicated in $\mathcal{U}$, when mixed boundary conditions are imposed via $\phi_{B, \Sigma_{1}}$. The remaining mappings are differential operator mappings, expressed weakly. $V$ acts from $\mathcal{U}$ to the dual of $\mathcal{H}_{0}^{s}$. For $\vec{\psi}$ in $\mathcal{H}_{0}^{s}$, this can be written in terms of the standard $L^{2}$ vector inner product as follows.

$$
V(\vec{v}, \vec{w}, n, p, \vec{\psi})=\nu_{*}(\nabla \vec{v}, \nabla \vec{\psi})_{L^{2}}+(\vec{v} \cdot \nabla \vec{w}, \vec{\psi})_{L^{2}}+(e / \rho)((p-n) \nabla \phi, \vec{\psi})_{L^{2}}
$$

The mappings $N, P$ are each defined from $\mathcal{U}$ into the dual of $H_{0, \Sigma_{1}}^{s}$. For $\omega_{n}, \omega_{p}$ in $H_{0, \Sigma_{1}}^{s}$, the explicit mappings are then specified:

$$
\begin{aligned}
& N\left(\vec{v}, \vec{w}, n, p, \omega_{n}\right)=\frac{1}{e}\left(\vec{J}_{n}, \nabla \omega_{n}\right)_{L^{2}}+\left(R_{n}, \omega_{n}\right)_{L^{2}}, \\
& P\left(\vec{v}, \vec{w}, n, p, \omega_{p}\right)=-\frac{1}{e}\left(\overrightarrow{J_{p}}, \nabla \omega_{p}\right)_{L^{2}}+\left(R_{p}, \omega_{p}\right)_{L^{2}} .
\end{aligned}
$$

Subsequent analysis will show that these mappings are well-defined. It is clear that $U$, with domain $\mathcal{U}$ restricted by (9), serves to define the system for the initial/boundary value problem as it evolves from $\vec{u}_{0}$ according to (10).

\subsection{The Semi-discrete Equations: Rothe's Method}

The technique of proof used for the fluid-transport system is Rothe's method

of horizontal lines. We will utilize the mappings just defined. Thus, for a specified $\Delta t=T / K$, we employ the following replacements for $1 \leq k \leq K$ :

$$
\frac{d \vec{v}}{d t} \mapsto \frac{\vec{v}_{k}-\vec{v}_{k-1}}{\Delta t}, \frac{d n}{d t} \mapsto \frac{n_{k}-n_{k-1}}{\Delta t}, \frac{d p}{d t} \mapsto \frac{p_{k}-p_{k-1}}{\Delta t}
$$

This leads to the following semi-discrete system:

$$
\begin{gathered}
\frac{\vec{v}_{k}-\vec{v}_{k-1}}{\Delta t}+V\left(\vec{v}_{k}, \vec{v}_{k}, n_{k}, p_{k}, \cdot\right)=0, \\
\frac{n_{k}-n_{k-1}}{\Delta t}+N\left(\vec{v}_{k}, \vec{v}_{k}, n_{k}, p_{k}, \cdot\right)=0, \\
\frac{p_{k}-p_{k-1}}{\Delta t}+P\left(\vec{v}_{k}, \vec{v}_{k}, n_{k}, p_{k}, \cdot\right)=0, \\
\nabla \cdot\left(\epsilon \nabla \phi_{k}\right)=e\left(n_{k}-p_{k}-d_{k}\right) .
\end{gathered}
$$

Here, $d_{k}$ is the obvious trace of $d$ on $t_{k}=k \Delta t$. Adjoined to these semidiscrete equations are the traces of the prescribed boundary conditions. We now examine the theory for these boundary value problems. 


\section{Existence Theory for Stationary Systems}

The analysis is motivated by the framework described in the monograph [11]. It is based upon finite-dimensional approximations, combined with an appropriate passage to the limit.

\subsection{The Framework and Abstract Stationary Problem}

Let $X, Y$ be separable, reflexive real Banach spaces, with $Y$ a subspace, densely and continuously embedded in $X$. We also suppose that $X$ is compactly embedded in the real reflexive Banach space $W$. In addition, we consider a mapping, $A: X \mapsto Y^{*}$, given explicitly by

$$
A(u)=L u+a(u, u, \cdot)+F(u, \cdot)
$$

where $L: X \mapsto X^{*}$ is an isomorphism, The structure of $L$ is induced by a continuous, coercive bilinear form $B(\cdot, \cdot)$ on $X \times X$ :

$$
\langle L u, v\rangle=B(u, v), B(u, u) \geq c\|u\|_{X}^{2}
$$

We outline the general assumptions now. In addition to the stated hypotheses, we assume:

(1) $a$ is continuous on $X \times X \times Y$ and $F$ is continuous on $X \times Y$.

(2) For each $u \in X, a(u, u, \cdot), F(u, \cdot)$ are continuous linear functionals on $Y$.

(3) The coerciveness property,

$$
\langle A(u), u\rangle /\|u\|_{X} \rightarrow \infty, \text { as }\|u\|_{X} \rightarrow \infty, u \in Y
$$

holds in the norm on $X$ for elements in $Y$.

(4) If $u_{k} \rightarrow u$ (weakly) in $X$ and $u_{k} \rightarrow u$ in $W$, then

$$
\begin{gathered}
a\left(u_{k}, u_{k}, v\right) \rightarrow a(u, u, v), \forall v \in Y, \\
F\left(u_{k}, v\right) \rightarrow F(u, v), \forall v \in Y .
\end{gathered}
$$

Theorem 3.1 Under the stated hypotheses, there is an element $u \in X$ satisfying $A(u)=f_{\mid Y}$ for a prescribed $f \in X^{*}$.

Proof The proof is a generalization of that described in [11]. At the outset, we select $r>0$ such that

$$
\langle A(u), u\rangle /\|u\|_{X} \geq\|f\|_{X^{*}}, \quad \forall\|u\|_{X}=r \text { and } u \in Y \text {. }
$$


This is possible by the third assumption. Using the separability hypothesis, we select a sequence $\left\{Y_{k}\right\}$ of increasing finite dimensional subspaces of $Y$, such that

$$
\cup_{k=1}^{\infty} Y_{k} \text { is dense in } Y .
$$

We now define subspaces $X_{k}$ of $X$, which agree with $Y_{k}$ as sets, but are imbued with the topology of $X$. We consider the finite dimensional problem of determining $u_{k} \in X_{k}$ such that

$$
A\left(u_{k}\right)=f_{\mid X_{k}} .
$$

By the continuity assumption, the restriction of $A$ is continuous on $X_{k}$, hence is pseudo-monotone [2, Prop. 21, p. 132]. By condition (13), we conclude that [11, Corollary 3.1.6] a solution $u_{k}$ exists, with $\left\|u_{k}\right\|_{X} \leq r$. Since $X$ is reflexive, and compactly embedded in $W$, we may extract a subsequence, which converges weakly in $X$, and strongly in $W$, to $u \in X$. To see that such an element $u$ is a solution of

$$
A(u)=f_{\mid Y},
$$

for prescribed $f \in X^{*}$, let $v \in Y$ and let $\varepsilon>0$ be prescribed. Choose $v_{\varepsilon} \in Y_{k(\varepsilon)}$ such that

$$
\left|\left\langle A(u)-f, v-v_{\varepsilon}\right\rangle\right|<\varepsilon / 2,
$$

which is possible by the denseness assumption applied to the $\left\{Y_{k}\right\}$, and by the second assumption. For $j \geq k(\varepsilon)$, we have, by the nestedness assumption of these spaces, and the definition of $u_{j}$,

$$
\left\langle A(u)-f, v_{\varepsilon}\right\rangle=\left\langle A(u)-A\left(u_{j}\right), v_{\varepsilon}\right\rangle,
$$

and, by the fourth assumption, $j$ can be selected so that this term is less than $\varepsilon / 2$ in absolute value. We conclude by the triangle inequality that

$$
|\langle A(u)-f, v\rangle|<\varepsilon .
$$

Since $\varepsilon$ is arbitrary, it follows that (14) holds. This completes the proof.

\subsection{The Existence Theory for the Semi-discrete Problems}

The functional analytic framework of the previous subsection is utilized here in our analysis of the semi-discrete problems defined by Rothe's method in $\S 2.2$. We now systematically make the identifications between the general existence theory of the previous section, and the semi-discrete problems. We first make an explanatory comment regarding the handling of boundary conditions. In order to maintain congruence with the existence theory, we shall write:

$$
\vec{v}=\vec{v}_{B}+\vec{\sigma}, n=n_{B}+\nu, p=p_{B}+\pi,
$$


where $\vec{\sigma}, \nu, \pi$ have zero boundary trace. This is made clear in the following subsection. However, in the actual verification of the continuity and coerciveness properties to follow, it is sometimes logically equivalent to consider $\vec{v}, n, p$, so that we adhere to this notationally simpler approach whenever possible.

\subsubsection{Identification of Function Spaces and Mappings}

The space $X$ is an adaptation of the domain $\mathcal{U}$ of the operator $U$ discussed in $\S 2.1$. Specifically, we require the finite energy spaces to be constrained further by zero boundary trace on $\partial \Omega$ or on $\Sigma_{1}$ :

$$
X=\mathcal{H}_{0} \times H_{0, \Sigma_{1}}^{1} \times H_{0, \Sigma_{1}}^{1}
$$

Similarly, the space $Y$ is defined by

$$
Y=\mathcal{H}_{0}^{s} \times H_{0, \Sigma_{1}}^{s} \times H_{0, \Sigma_{1}}^{s}
$$

and $W$ is defined by

$$
W=W_{0} \times L^{2} \times L^{2}
$$

where $W_{0}=\prod_{1}^{m} L^{2}$.

- We note that the assumptions of $\S 3.1$ hold for these spaces.

We now make the identifications with the mappings of $\S 3.1$. Because of the definition of $X$ used here, where the homogeneous boundary conditions are employed, we necessarily carry this over to $B, a$, and $F$. Accordingly, we write:

$$
\vec{u}=(\vec{v}, n, p)=\left(\vec{v}_{B}+\vec{\sigma}, n_{B}+\nu, p_{B}+\pi\right)
$$

and formulate the definitions in terms of $\vec{\zeta}=(\vec{\sigma}, \nu, \pi)$.

- We begin with $B(\cdot, \cdot)$ :

$$
\begin{gathered}
B(\vec{\zeta}, \vec{\zeta})=\nu_{*}(\nabla \vec{\sigma}, \nabla \vec{\sigma})_{L^{2}}+\Delta t^{-1}(\vec{\sigma}, \vec{\sigma})_{L^{2}}+D_{n}(\nabla \nu, \nabla \nu)_{L^{2}}+\Delta t^{-1}(\nu, \nu)_{L^{2}} \\
+D_{p}(\nabla \pi, \nabla \pi)_{L^{2}}+\Delta t^{-1}(\pi, \pi)_{L^{2}}
\end{gathered}
$$

- Furthermore, the functional $a(\cdot, \cdot, \cdot)$ is given concretely by

$$
a(\vec{\sigma}, \vec{\tau}, \cdot)=\left(\left(\vec{v}_{B}+\vec{\sigma}\right) \cdot \nabla\left(\vec{v}_{B}+\vec{\tau}\right), \cdot\right)_{L^{2}}=(\vec{v} \cdot \nabla \vec{w}, \cdot)_{L^{2}}
$$

For later reference, we define: $b(\vec{v}, \vec{w}, \cdot):=a(\vec{\sigma}, \vec{\tau}, \cdot)$.

- Finally, we turn to the identification of $F$. The structure of the proof is such that we are required to define a certain modification of a direct transcription 
of the equations. This modification requires the truncation operator:

$$
\tau_{M} f(x)=\left\{\begin{array}{cc}
f(x), & \text { for }-M \leq f(x) \leq M \\
\operatorname{signum}(f(x)) M, & \text { otherwise }
\end{array}\right.
$$

where $f$ is a measurable real function. Its definition may be extended to a vector function componentwise. $\tau_{M}$ is Lipschitz continuous on $L^{2}$. Thus, we define, in terms of $\vec{v}, n, p$ :

$$
\begin{gathered}
F_{M}\left(\vec{v}, n, p ; \vec{\psi}, \omega_{n}, \omega_{p}\right)=(e / \rho)\left(\left(\tau_{M}(p)-\tau_{M}(n)\right) \nabla \phi, \vec{\psi}\right)_{L^{2}} \\
-c_{n}\left(\tau_{M}(n) \nabla \phi, \nabla \omega_{n}\right)_{L^{2}}-\left(n \tau_{M}(\vec{v}), \nabla \omega_{n}\right)_{L^{2}}+c_{p}\left(\tau_{M}(p) \nabla \phi, \nabla \omega_{p}\right)_{L^{2}} \\
-\left(p \tau_{M}(\vec{v}), \nabla \omega_{p}\right)_{L^{2}}+\left(R_{n}(\vec{v}, n, p), \omega_{n}\right)_{L^{2}}+\left(R_{p}(\vec{v}, n, p), \omega_{p}\right)_{L^{2}} .
\end{gathered}
$$

Here, we have used the relations: $c_{n}=\frac{e D_{n}}{k T_{0}}, c_{p}=\frac{e D_{p}}{k T_{0}}$.

- Finally, the right hand side element $f$ is given by

$$
\begin{gathered}
f\left(\vec{\psi}, \omega_{n}, \omega_{p}\right)=-\nu_{*}\left(\nabla \vec{v}_{B}, \nabla \vec{\psi}\right)_{L^{2}}-D_{n}\left(\nabla n_{B}, \nabla \omega_{n}\right)_{L^{2}}-D_{p}\left(\nabla p_{B}, \nabla \omega_{p}\right)_{L^{2}} \\
+\Delta t^{-1}\left\langle\vec{u}_{k-1}, \vec{\psi}\right\rangle+\Delta t^{-1}\left\langle n_{k-1}, \omega_{n}\right\rangle+\Delta t^{-1}\left\langle p_{k-1}, \omega_{p}\right\rangle \\
-\Delta t^{-1}\left\langle\vec{u}_{B}\left(t_{k}\right), \vec{\psi}\right\rangle-\Delta t^{-1}\left\langle n_{B}\left(t_{k}\right), \omega_{n}\right\rangle-\Delta t^{-1}\left\langle p_{B}\left(t_{k}\right), \omega_{p}\right\rangle .
\end{gathered}
$$

It is understood that $\phi$ is implicitly defined via the Poisson equation (6).

\subsubsection{Continuity Properties}

The analysis of the bilinear form $B$ is standard. The properties of the functional $a$ are now discussed. Joint continuity in the argument $(\vec{\sigma}, \vec{\tau}, \vec{\psi})$ (i. e. , on $X \times X \times Y)$ is equivalent to continuity of $b$ in the argument $(\vec{v}, \vec{w}, \vec{\psi})$; continuity in the latter argument is a consequence of the generalized Hölder inequality:

$$
|a(\vec{\sigma}, \vec{\tau}, \vec{\psi})|=|b(\vec{v}, \vec{w}, \vec{\psi})| \leq C\|\vec{v}\|_{L^{2}}\|\vec{w}\|_{H^{1}}\|\vec{\psi}\|_{H^{s}}
$$

for $s \geq m / 2$. This estimate first uses $b(\vec{v}, \vec{w}, \vec{\psi})=-b(\vec{v}, \vec{\psi}, \vec{w})$, followed by the Hölder and Sobolev inequalities (see $[26,11])$. For the former inequality, the reciprocal indices are $1 / 2,1 / m,(m-2) /(2 m)$, resp. Note that $s \geq m / 2$ implies that $H^{s-1}$ is continuously embedded into $L^{m}$. Continuity is established via the triangle inequality. We now consider the functional $F_{M}$, and estimate the individual terms for continuity via a generalized Hölder inequality.

(1) We see that the functional,

$$
(e / \rho)\left(\left(\tau_{M}(p)-\tau_{M}(n)\right) \nabla \phi, \vec{\psi}\right)_{L^{2}}
$$


satisfies the estimate,

$$
\left|(e / \rho)\left(\left(\tau_{M}(p)-\tau_{M}(n)\right) \nabla \phi, \vec{\psi}\right)_{L^{2}}\right| \leq C\left\|\tau_{M}(p)-\tau_{M}(n)\right\|_{L^{2}}\|\phi\|_{H^{2}}\|\vec{\psi}\|_{H^{s-1}}
$$

This is sufficient to infer continuity of this term on $X \times Y$.

(2) The terms, $-c_{n}\left(\tau_{M}(n) \nabla \phi, \nabla \omega_{n}\right)_{L^{2}}$ and $c_{p}\left(\tau_{M}(p) \nabla \phi, \nabla \omega_{p}\right)_{L^{2}}$, make use of the estimate:

$$
\left|c_{n}\left(\tau_{M}(n) \nabla \phi, \nabla \omega_{n}\right)_{L^{2}}\right| \leq C\left\|\tau_{M}(n)\right\|_{L^{2}}\|\phi\|_{H^{2}}\left\|\omega_{n}\right\|_{H^{s}}
$$

and a similar estimate with $n$ replaced by $p$. The reasoning proceeds as before, via (16).

(3) The terms, $-\left(n \tau_{M}(\vec{v}), \nabla \omega_{n}\right)_{L^{2}}$ and $-\left(p \tau_{M}(\vec{v}), \nabla \omega_{p}\right)_{L^{2}}$, make use of

$$
\left|\left(n \tau_{M}(\vec{v}), \nabla \omega_{n}\right)_{L^{2}}\right| \leq C\|n\|_{H^{1}}\left\|\tau_{M}(\vec{v})\right\|_{L^{2}}\left\|\omega_{n}\right\|_{H^{s}}
$$

and a similar estimate with $n$ replaced by $p$. Continuity follows as above.

Since the terms involving $R_{n}, R_{p}$ are routinely continuous by the Nemytskii property, we have proven that $F_{M}$ is continuous on $X \times Y$.

\subsubsection{Coerciveness}

The bilinear form $B$ is coercive by hypothesis. We consider separately the functionals $a$ and $F_{M}$.

- We begin with $a$.

In this case, we observe: $a(\vec{\sigma}, \vec{\sigma}, \vec{\sigma})=b\left(\vec{v}, \vec{v}, \vec{v}-\vec{v}_{B}\right)$, and we estimate:

$$
b\left(\vec{v}, \vec{v}, \vec{v}-\vec{v}_{B}\right)=b(\vec{v}, \vec{v}, \vec{v})-b\left(\vec{v}, \vec{v}, \vec{v}_{B}\right) \geq-C\|\vec{v}\|_{L^{2}}\|\vec{v}\|_{\mathcal{H}}\left\|\vec{v}_{B}\right\|_{L^{\infty}}
$$

Notice that the inequality:

$$
b(\vec{v}, \vec{v}, \vec{v}) \geq 0
$$

has been used. This follows from:

$$
b(\vec{v}, \vec{v}, \vec{v})=\frac{1}{2} \int_{\Omega} \vec{v} \cdot \nabla|\vec{v}|^{2} d x=\frac{1}{2} \int_{\Omega} \nabla \cdot\left(\vec{v}|\vec{v}|^{2}\right) d x,
$$

which is then integrated by parts, and observed to have a nonnegative boundary integral by the assumption on the sign of the normal boundary component of $\vec{v}_{B}$. Notice that, prior to integration by parts, we have used the divergence free property of $\vec{v}$. In order to analyze (18), one uses the usual product bound in terms of sums of squares, of the form $\alpha \beta \leq \frac{1}{2}\left(\delta \alpha^{2}+\delta^{-1} \beta^{2}\right)$, followed by the decomposition of $\vec{v}$, in terms of boundary traces. The homogeneous trace terms may be absorbed into $B$ for $\Delta t$ sufficiently small; the remaining terms are controlled by problem dependent constants. 
- We now turn to $F_{M}$.

(1) Each of the terms:

$$
\begin{gathered}
(e / \rho)\left(\left(\tau_{M}(p)-\tau_{M}(n)\right) \nabla \phi, \vec{\psi}\right)_{L^{2}},-c_{n}\left(\tau_{M}(n) \nabla \phi, \nabla \nu\right)_{L^{2}}, \\
c_{p}\left(\tau_{M}(p) \nabla \phi, \nabla \pi\right)_{L^{2}},-\left(n \tau_{M}(\vec{v}), \nabla \nu\right)_{L^{2}},-\left(p \tau_{M}(\vec{v}), \nabla \pi\right)_{L^{2}},
\end{gathered}
$$

is handled by the technique used for (18). Note that the intermediate bounding of $\|\nabla \phi\|_{L^{2}}$ in terms of the $L^{2}$ norms of $n, p, d$ is first required.

(2) The remaining two terms in the definition of $F_{M}$ can be analyzed in a manner similar to the preceding terms because of the boundedness assumptions for $R_{n}, R_{p}$. Altogether, the coerciveness follows.

\subsubsection{Sequential Convergence}

Assumption (4) of $\S 3.1$ is verified as follows. The convergence result for $a$ has been discussed in [26]. The terms in $F_{M}$ involving truncation are special cases of Lemma 3.1 of [15]. The Nemytskii property implies a similar result for the terms involving $R_{n}, R_{p}$. This completes the hypothesis verification.

\subsubsection{The Major Theorem for the Stationary Problem}

We now are prepared to state the desired result. The proof has already been provided in the previous subsections. We note that the element $f$ as defined above can be extended to be a continuous linear functional on $X$.

Theorem 3.2 If $\left\langle A_{M} u, \cdot\right\rangle$ is defined as the sum $B(u, \cdot)+a(u, u, \cdot)+\left\langle F_{M} u, \cdot\right\rangle$, we conclude the existence of a solution of the equation: $A_{M}(u)=f_{\mid Y}$ for the above prescribed $f \in X^{*}$, provided $\Delta t$ is sufficiently small. In particular, solutions exist for the boundary value problems defined in §2.2.

\subsection{Estimates for the Semidiscrete Approximations}

We now turn our attention to the derivation of bounds for the semi-discrete solutions, whose existence has been established in the previous subsection. In order to maintain consistency in notation, we employ:

$$
\vec{u}_{k, \Delta t}=\left(\vec{v}_{k, \Delta t}, n_{k, \Delta t}, p_{k, \Delta t}\right)
$$

for the semi-discrete solutions at level $t=k \Delta t$. We will derive estimates of the following form. 
Lemma 3.1 There are positive constants $\alpha, \beta, \gamma$, and $C$, which do not depend on $M$, but only on the fixed charge $d$, and the initial and boundary data, such that, for each $1 \leq k \leq K$ :

$$
\alpha\left\|\vec{u}_{k, \Delta t}\right\|_{L^{2}}^{2}+\beta \sum_{j=1}^{j=k}\left\|\vec{u}_{j, \Delta t}-\vec{u}_{j-1, \Delta t}\right\|_{L^{2}}^{2}+\gamma \sum_{j=1}^{j=k}\left\|\nabla \vec{u}_{j, \Delta t}\right\|_{L^{2}}^{2} \Delta t \leq C .
$$

The dependence is on the $L^{2}$ norm of $\vec{u}_{0}$; the norm in $C\left([0, T] ; H^{1}(\Omega)\right) \cap$ $H^{1}\left((0, T) ; L^{2}(\Omega)\right)$ of the components of $\vec{u}_{B}$; and the norms of $d$ and the components of $\vec{v}_{B}$ in $C\left([0, T] ; L^{2}(\Omega)\right)$ and $C\left([0, T] ; H^{s}(\Omega)\right)$, resp.

Proof For notational simplicity, we suppress the subscript $\Delta t$; thus we write $\vec{u}_{k}=\left(\vec{v}_{k}, n_{k}, p_{k}\right)$. We first note that the usual way of proceeding, by choosing a test function from a finite energy space, directly related to the solution, is not valid, since the equation of Theorem 3.2 is distributional. The argument of how to circumvent this limitation is carried out in [11, Remark 3.3.4], which involves a passage to the limit with respect to the smooth finite dimensional subspaces and the use of lower semicontinuity with respect to weak convergence. The present situation is slightly more complicated because of inhomogeneous boundary conditions. The approach is to choose projections of the components of $\vec{u}_{k}-\vec{u}_{B}\left(t_{k}\right)$ onto the nested smooth subspaces, and estimate the limit infimum, just as in [11]. By isolating the divided difference and gradient terms on the left hand side, and exploiting the identity,

$$
\left(\vec{v}_{j}-\vec{v}_{j-1}, \vec{v}_{j}\right)_{L^{2}}=(1 / 2)\left\{\left\|\vec{v}_{j}\right\|^{2}+\left\|\vec{v}_{j}-\vec{v}_{j-1}\right\|^{2}-\left\|\vec{v}_{j-1}\right\|^{2}\right\}
$$

with similar identities for the other variables, one obtains, after addition on $j=1, \ldots, k$, the left hand side of the inequality, perturbed by initial value norms. The right hand side is obtained by standard estimation. This uses summation by parts for the boundary value functions, and the properties of $b$ and the $\phi$-inversion. We omit the details in the interest of brevity. $\square$.

\section{Weak Solutions of the Evolution System}

The following theorem is proven. It is the core result of this article.

Theorem 4.1 There is a weak solution in the sense of Definition 1.1.

Remark The theorem holds for the PNP/Stokes system without the outward flux boundary assumption.

A two-step procedure is required here: 
- Establishing a weak solution relation, as in Definition 1.1, with truncation. The following subsection formulates this precisely.

- Justification of a further limit to eliminate truncation.

Before proceeding, we interpret the spaces in conformity with the operator $U$ of $\S 2$; thus, $X=\mathcal{H} \times H^{1} \times H^{1}$, and

$$
Y=\mathcal{H}_{0}^{s} \times H_{0, \Sigma_{1}}^{s} \times H_{0, \Sigma_{1}}^{s}
$$

For the two steps indicated above, we select test functions satisfying:

$$
\left(\vec{\psi}, \omega_{n}, \omega_{p}\right) \in C^{\infty}(\overline{\mathcal{D}}), \quad\left(\vec{\psi}(\cdot, t), \omega_{n}(\cdot, t), \omega_{p}(\cdot, t)\right) \in Y, 0 \leq t \leq T .
$$

A routine limit at the conclusion gives the result for the test function class in Definition 1.1. The first stage of the analysis is now defined.

\subsection{Weak Solutions for the Modified System}

We rewrite the modification of Definition 1.1 for clarity, with truncation incorporated.

$$
\begin{aligned}
& \int_{\mathcal{D}}\left[\rho \vec{v} \frac{\partial \vec{\psi}}{\partial t}-\eta \nabla \vec{v} \cdot \nabla \vec{\psi}\right] d \xi d t-\int_{0}^{T} \rho a(\vec{v}, \vec{v}, \vec{\psi}) d t \\
& -e \int_{\mathcal{D}}\left(\tau_{M}(p)-\tau_{M}(n)\right) \nabla \phi \cdot \vec{\psi} d \xi d t=\int_{\Omega \times\{T\}} \vec{v} \cdot \vec{\psi} d \xi-\int_{\Omega \times\{0\}} \overrightarrow{v_{0}} \cdot \vec{\psi} d \xi \\
& \int_{\mathcal{D}}\left[n \frac{\partial \omega_{n}}{\partial t}-D_{n} \nabla n \cdot \nabla \omega_{n}+\left(\frac{e D_{n}}{k T_{0}}\right) \tau_{M}(n) \nabla \phi \cdot \nabla \omega_{n}+\tau_{M}(\vec{v}) n \cdot \nabla \omega_{n}\right] d \xi d t \\
& =\int_{\Omega \times\{T\}} n \omega_{n} d \xi-\int_{\Omega \times\{0\}} n_{0} \omega_{n} d \xi+\int_{\mathcal{D}} R_{n} \omega_{p} d \xi d t \\
& \int_{\mathcal{D}}\left[p \frac{\partial \omega_{p}}{\partial t}-D_{p} \nabla p \cdot \nabla \omega_{p}-\left(\frac{e D_{p}}{k T_{0}}\right) \tau_{M}(p) \nabla \phi \cdot \nabla \omega_{p}+\tau_{M}(\vec{v}) p \cdot \nabla \omega_{p}\right] d \xi d t \\
& =\int_{\Omega \times\{T\}} p \omega_{p} d \xi-\int_{\Omega \times\{0\}} p_{0} \omega_{p} d \xi+\int_{\mathcal{D}} R_{p} \omega_{n} d \xi d t
\end{aligned}
$$

\subsubsection{Step Function and Piecewise Linear Approximation Sequences}

Throughout this subsection, all solutions correspond to semidiscrete solutions defined by $A_{M}$. The dependence on $M$, via $F_{M}$, is understood, but not explicitly stated. $M$ is fixed here. We now define the sequences which permit compactness estimates. Indeed, $\vec{u}_{S, \Delta t}$ is the step function and $\vec{u}_{P L, \Delta t}$ the piecewise linear interpolant, both defined by the semidiscrete solutions. Explicitly, for each $1 \leq k \leq K$, and

$$
(k-1) \Delta t \leq t<k \Delta t
$$


define

$$
\vec{u}_{S, \Delta t}(\cdot, t)=\vec{u}_{k, \Delta t}, \vec{u}_{P L, \Delta t}(\cdot, t)=\vec{u}_{k, \Delta t}+\frac{(t-k \Delta t)\left(\vec{u}_{k, \Delta t}-\vec{u}_{k-1, \Delta t}\right)}{\Delta t},
$$

We have the following estimates.

Lemma 4.1 The sequences, $\vec{u}_{S, \Delta t}(x, t), \vec{u}_{P L, \Delta t}(x, t)$, are bounded in the topology of $L^{2}((0, T) ; X)$, and $\vec{u}_{P L, \Delta t}(x, t)$ is bounded in $H^{1}\left((0, T) ; Y^{*}\right)$. The bounds do not depend on the truncation parameter $M$.

Proof One uses the bounds derived in (19) to obtain the result that the sequences are bounded in $L^{2}((0, T) ; X)$. Direct estimation, equation by equation, is required for the result that $\vec{u}_{P L, \Delta t}(x, t)$ is bounded in $H^{1}\left((0, T) ; Y^{*}\right)$. The estimate (for each $1 \leq k \leq K$ ):

$$
\sum_{j=1}^{j=k}\left\|\vec{u}_{j, \Delta t}-\vec{u}_{j-1, \Delta t}\right\|_{Y^{*}}^{2}(1 / \Delta t) \leq C
$$

suffices. For the first equation of the system (11), involving $\vec{v}$, direct estimation uses the bound:

$$
|b(\vec{v}, \vec{v}, \vec{\psi})| \leq C\|\vec{v}\|_{L^{2}}\|\vec{v}\|_{H^{1}}, \text { for }\|\vec{\psi}\|_{\mathcal{H}^{s}} \leq 1
$$

The use of (19) provides a uniform $L^{2}$ bound, hence a justification for the estimate

$$
\sum_{j}\left|b\left(\vec{v}_{j, \Delta t}, \vec{v}_{j, \Delta t}, \vec{\psi}\right)\right|^{2} \Delta t \leq C .
$$

Again, the use of (19) allows the direct estimation of the other term in the Navier-Stokes contribution to (21). In particular, because of the $H^{2}$ regularity assumed for the Poisson inversion map, we conclude that $\nabla \phi_{k}$ is uniformly $H^{1}$ bounded. This is used for the forcing term; we do not make use of the truncation here, but use the Hölder inequality, followed by the Sobolev inequality, as previously. For the contributions given by the equations involving $n, p$, one must estimate:

$$
\left(\frac{e D_{n}}{k T_{0}}\right)^{2} \sum_{j}\left|\left(\tau_{M}\left(n_{j}\right) \nabla \phi_{j}, \nabla \omega_{n}\right)_{L^{2}}\right|^{2} \Delta t
$$

with $\omega_{n}$ bounded in $H^{s}$ norm. By (19), and the regularity assumed for the Poisson inversion map, we have an invariant bound. This leaves the term:

$$
\sum_{j}\left|\left(n_{j} \tau_{M}\left(\vec{v}_{j}\right), \nabla \omega_{n}\right)_{L^{2}}\right|^{2} \Delta t
$$

which is analyzed by Hölder's inequality (followed by the Sobolev inequality). The upper bound is proportional to $\sum_{j}\left\|\vec{v}_{j}\right\|_{\mathcal{H}}^{2} \Delta t$, already estimated in (19). The $p$-terms are analogous. 


\subsubsection{Weak Solutions of the Reduced System: Convergent Subsequences}

Lemma 4.2 Given the modification of Definition 1.1 induced by truncation, as defined in $\S 4.1$, and given the $C^{\infty}$ test functions introduced above, a weak solution $\vec{u}=\vec{u}^{M}$ exists for each fixed $M$.

Proof The details are given in the proof of [11, Theorem 5.4.1]. The convergence for additional terms not included in [11] follows the lines of the final lemma in [15]. The critical use of the derived inequalities is to extract weakly convergent subsequences (corresponding to $\left.(\Delta t)_{\ell}=T / K_{\ell}\right)$ in $L^{2}((0, T) ; X)$ : $\vec{u}_{S, \ell}(x, t), \vec{u}_{P L, \ell}(x, t)$. By use of Lemma 4.1 , and the subsequent application of the Aubin lemma [11, Lemma 5.4.2], one infers that these sequences have a common limit. In fact, a further subsequence of $\vec{u}_{P L, \ell}(x, t)$ is actually convergent in $L^{2}(\mathcal{D})$. One also sees that the corresponding $\tau_{M}$ truncations have the same property. By combining averages of test functions with integrals of the step function sequence, one obtains the pre-relations whose limit defines the weak solution. A separate calculation, making use of the time derivative properties of $\vec{u}_{B}$, yields the boundary conditions for the limit $\vec{u}$.

\subsection{Conclusion of the Proof of Theorem 4.1}

It remains to conclude the proof of Theorem 4.1. In this section, we build upon the restricted weak solutions of Definition 1.1, as presented in Lemma 4.2 , and discuss the limit as $M \rightarrow \infty$. Each solution $\vec{u}^{M}$ in the sense of Lemma 4.2 satisfies the bounds of Lemma 4.1, and these are independent of $M$. Since the test functions at this point are essentially bounded, one can take subsequential limits in the equations of the modified system to obtain the unrestricted equations of Definition 1.1; a final limit gives the test functions in the general class.

Remark. The question arises as to whether $n, p$ can be chosen to be nonnegative. One can implement this argument at the level of the semidiscrete equations (11), via a discrete Gronwall inequality, as derived in [11]. Notice that the reasoning used in [12, Proposition 2.1] is not directly valid here, since the time derivatives of $n, p$ are distributions. However, an appropriate set of arguments may be employed, as applied to (11), since the negative parts of $n, p$ are members of $H_{0, \Sigma_{1}}^{1}$, and may be (approximated by) test functions. Initial and boundary values are required to be nonnegative in this case, for the validity of the argument via the discrete Gronwall inequality. Altogether, one obtains nonnegativity for the modified system, and, finally, for the system of

Definition 1.1. 


\section{Computational Aspects and Perspectives}

In this concluding section, we briefly discuss the main relevant issues concerning the numerical treatment of the PNP/NS system, and its application in the modeling of several realistic problems arising in Bio-Engineering.

\subsection{Numerical Approximation}

A staggered algorithm is adopted for the successive solution of the PNP and NS subsystems, in the same fashion as in the treatment of fluid-structure interaction problems. For each time level $t_{k}$, a PNP system with a given velocity field $\mathbf{v}^{(k)}$ is solved using the Gummel Map typically employed in semiconductor device simulation [13]. This provides in output the updated concentrations $n^{(k+1)}, p^{(k+1)}$ and electric field $\mathbf{E}^{(k+1)}$. Then, the NS system is solved using a fixed point iteration based on Oseen subproblems [22]. This provides in out-

put the updated velocity $\mathbf{v}^{(k+1)}$ and pressure $P_{f}^{(k+1)}$. The process is repeated until self-consistency is achieved for the solution at the considered time level. The numerical discretization of the linearized subproblems is carried out using (stabilized) dual-mixed hybridized finite element formulations [4,3]. This ensures the same computational accuracy for scalar and vector/tensor-valued unknowns, local conservation and self-equilibrium, and nonnegativity of concentrations. In the case of the continuity equations in the linearized PNP system, exponential fitting is included in the scheme to deal with the presence of highly dominating advective terms. In the case of the momentum balance equation in the linearized NS system, a Discontinuous Galerkin upwind treatment is introduced for stabilization.

\subsection{Applications}

Contemporary applications in Bio-Engineering are characterized by highly sophisticated interacting systems, as in Lab-On-Chip technology, where electrochemical and fluid-mechanical transport of substances, chemical reactions and electrical transduction of biological signals simultaneously occur, at strongly varying spatial and temporal scales. Significant examples of such systems, for which the PNP/NS model investigated in this article provides an appropriate description in most relevant situations, are:

- Bio-chips for neuronal cell monitoring. The basic configuration of such biohybrid devices consists of a cell in an electrolyte solution, that is grown onto the surface of an electronic substrate with the aim of transducing an ionic current signal into an electric output current. Bio-chips encompass 
significant spatial and temporal scales. For example, the ion channels in the cell membrane are nanometers in length, and the current is gauged on the nanosecond scale. The channel gating is on the millisecond scale. The cell is of micron dimensions, and the communication between cell and transistor is affected by gating. We refer to [9] for a thorough description of the system and to [21] for further details on the characterization of the neuronal/transistor interface.

- Bio-reactors for cell growth in tissue engineering. The basic configuration of such devices is a three-layer structure including two fluid layers and, in between, a porous scaffolded bio-compatible matrix where cells are grown upon hydrodynamical perfusion of a nutrient culture medium. Again, the device encompasses significant spatial and temporal scales, the scaffold being of centimeter dimensions, and the single pore being of micron dimensions, while a typical culture period is of the order of weeks. We refer to [23,7] for a detailed description of the bio-reactor architecture and of the analysis of the role of shear stress and perfusion rate on the effectiveness of the culture procedure. A recent example of mathematical modeling and numerical simulation of bio-reactors can also be found in [6].

- Microfluidic chambers in Lab-On-Chip technology. These devices are frequently used in micro-electro-mechanical systems (MEMS) in the chemical and bio-medical industry, or in electro-kinetic transport processes such as electro-osmosis and electro-phoresis. A mathematical model similar to the one discussed in this article, together with extensive numerical investigation, is presented in $[5,16]$.

Acknowledgment: The authors gratefully acknowledge Dr. Bice Chini (CNR Istituto di Neuroscienze Cellulari e Sezione di Farmacologia Molecolare, Milano Italy) for useful discussions and suggestions, and Ing. Massimo Longaretti (formerly intern fellow at Politecnico di Milano, Italy, presently at STMicroelectronics, Agrate Brianza, Italy) for his continuous effort and support in the research activity illustrated in Sect. 5.2.

\section{References}

[1] G. Avalos and R. Triggiani. Mathematical analysis of PDE systems which govern fluid-structure interactive phenomena. Bol. Soc. Parana. Mat. (3) 25 (2007), no. 1-2, 17-36.

[2] H. Brezis. Équations et inquations non linaires dans les espaces vectoriels en dualité. (French) Ann. Inst. Fourier (Grenoble) 18 (1968), 115-175.

[3] F. Brezzi, L.D. Marini, S. Micheletti, P. Pietra, and R. Sacco. Stability and error analysis of mixed finite volume methods for advective-diffusive problems. Comput. Math. Appl. 51 (2006), 681-696. 
[4] P. Causin and R. Sacco. A dual-mixed hybrid formulation for fluid mechanics problems: mathematical analysis and application to semiconductor process technology. Comput. Methods Appl. Mech. Engrg. 192 (2003), 593-612.

[5] H.-W. Choi and M. Paraschivoiu. Advanced hybrid-flux approach for output bounds of electro-osmotic flows: adaptive refinement and direct equilibrating strategies. Microfluidics and Nanofluidics 2 (2006), 154-170.

[6] C.A. Chung, C.W. Chen, C.P. Chen, and C.S. Tseung. Enhancement of cell growth in tissue engineering constructs under direct perfusion: modeling and simulation. Biotechnol. Bioeng. 97 (2007), 1603-1616.

[7] M. Cioffi, F. Boschetti, M.T. Raimondi, and G. Dubini. Modeling evaluation of the fluid-dynamic microenvironment in tissue-engineered constructs: a microCT based model. Biotechnol. Bioeng. 93 (2006), 500-510.

[8] B. Cockburn, G.E. Karniadakis, and C.-W. Shu. The development of Discontinuous Galerkin methods. In (B. Cockburn, G.E. Karniadakis, and C.W. Shu, eds.), Lecture Notes in Computational Science and Engineering 11, Springer New York, 2000, 3-50.

[9] P. Fromherz. Neuroelectronics interfacing: Semiconductor chips with ion channels, cells, and brain. In (R. Weise, ed.) Nanoelectronics and Information Technology, Wiley-VCH, Berlin, 2003, 781-810.

[10] S. Gala. Regularity criterion on weak solutions to the Navier-Stokes equations. J. Korean Math. Soc. 45 (2008), 537-558.

[11] J.W. Jerome. Approximation of Nonlinear Evolution Systems, Academic Press, 1983.

[12] J.W. Jerome. Evolution systems in semiconductor device modeling: A cyclic uncoupled line analysis for the Gummel map. Math. Meth. Appl. Sci. 9 (1987), $455-492$.

[13] J.W. Jerome, Analysis of Charge Transport, Springer-Verlag Berlin Heidelberg, 1996.

[14] J.W. Jerome. An analytical approach to charge transport in a moving medium. Transport Theory Statist. Phys. 31 (2002), 333-366.

[15] J.W. Jerome. A trapping principle and convergence result for finite element approximate solutions of steady reaction diffusion systems. Numer. Mathematik 109 (2008), 121-142.

[16] Y. Hu, J. Lee, C. Werner, and D. Li. Electrokinetically controlled concentration gradients in micro-chambers in microfluidic systems. Microfluidics and Nanofluidics 2 (2006), 141-153.

[17] M. Longaretti, G. Marino, B. Chini, J. Jerome, and R. Sacco. Computational models in nano-bio-electronics: simulation of ionic transport in voltage operated channels. Journal of Nanoscience and Nanotechnology 8 (2008), 3686-3694. 
[18] M. Longaretti, B. Chini, J. Jerome, and R. Sacco. Electrochemical modeling and characterization of voltage operated channels in nano-bio-electronics. Sensor Letters 6 (2008), 49-56.

[19] M. Longaretti, B. Chini, J. Jerome, and R. Sacco. Computational modeling and simulation of complex systems in bio-electronics. Journal of Computational Electronics 7 (2008), 10-13.

[20] M. Mitrea and S. Monniaux. The regularity of the Stokes operator and the Fujita-Kato approach to the Navier-Stokes initial value problem in Lipschitz domains. J. Funct. Anal. 254 (2008), 1522-1574.

[21] A. Offenhäuser, J. Rühe, and W. Knoll. Neuronal cells cultured on modified microelectronic device structures. Journal of Vacuum Science and Technology A 13 (5) (1995), 2606-2612.

[22] A. Quarteroni and A. Valli. Domain Decomposition Methods for Partial Differential Equations, Oxford Science Publications, New York (1999).

[23] M.T. Raimondi, F. Boschetti, F. Migliavacca, M. Cioffi, and G. Dubini. Microfluid dynamics in three-dimensional engineered cell systems in bioreactors. In (N. Ashammakhi and R.L. Reis, eds.), Topics in Tissue Engineering, Vol. 2, 2005, Chapter 9.

[24] I. Rubinstein. Electro-diffusion of Ions, SIAM, 1990.

[25] F. Saidi. On the Navier-Stokes equation with slip boundary conditions of friction type. Math. Model. Anal. 12 (2007), 389-398.

[26] R. Temam. Navier-Stokes Equations, North-Holland Publishers (3rd. rev. ed.), 1984. 